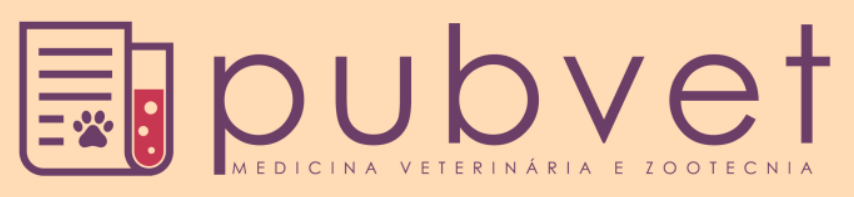

https://doi.org/10.22256/pubvet.v12n3a52.1-4

\title{
Ocorrência de giardia spp. em fezes de cães na praia do laranjal, Pelotas-RS e estudo comparativo entre técnicas
}

\author{
Tainá Ança Evaristo Mendes Cardoso ${ }^{\ominus}{ }^{1 *}$, Maysa Seibert de Leão ${ }^{1}$ Bruna dos Santos \\ Pires ${ }^{2}$, Tatiana de Ávila Antunes ${ }^{2}$, Diego Moscarelli Pinto ${ }^{\circ}{ }^{3}$, Leandro Quintana \\ Nizoli ${ }^{3}$, Alexsander Ferraz ${ }^{4}$ \\ ${ }^{I}$ Graduanda do curso de medicina veterinária da Universidade Federal de Pelotas (UFPel), Pelotas - RS, Brasil. \\ ${ }^{2}$ Médica Veterinária, Pelotas - RS, Brasil.Email:bruspires@gmail.com;tatdavila@bol.com.br \\ ${ }^{3}$ Professor Ajunto da Universidade Federal de Pelotas (UFPel), Departamento de Veterinária Preventiva, Capão do Leão RS, Brasil, \\ Email:dimoscarelli@yahoo.com.br; leandro.nizoli@gmail.com \\ ${ }^{4}$ Médico Veterinário, Residente Multiprofissional na área de Doenças e Zoonoses Parasitárias na Universidade Federal de Pelotas (UFPel), \\ Departamento de Veterinária Preventiva, Pelotas - RS, Brasil. Email: xanderferraz@yahoo.com.br \\ * Autor para correspondência, E-mail: evaristo.medvet@gmail.com
}

RESUMO. A giardiose é uma importante doença zoonótica causada por protozoários, facilmente disseminada pela ingestão de cistos na água e alimento. Para identificação parasitológica deste agente prioriza-se as técnicas laboratoriais coproparasitológicas, sendo algumas delas o método de Faust e a técnica de Hoffman, Pons e Janer (HPJ). O objetivo deste estudo foi avaliar amostras de fezes de cães, coletadas na orla da praia do Laranjal, no município de Pelotas, Rio Grande do Sul, Brasil, e comparar estas duas técnicas de diagnóstico, utilizadas na rotina laboratorial. Das 41 amostras analisadas por ambas as técnicas, nove foram positivas pelo método de Faust e sete pela de HPJ, representando 22\% e $17 \%$ respectivamente. Pelo fato de ser um local público e a giardiose apresentar potencial zoonótico, é necessário adotar medidas sanitárias que visem à diminuição da contaminação destes locais e consequentemente a exposição a este protozoário.

Palavras chave: comparação de técnicas, diagnóstico, giardiose, protozoário, zoonose

\section{Occurrence of giardia spp. in dog feces on the laranjal beach, Pelotas- RS and comparative study between techniques}

\begin{abstract}
Giardiosis is an important zoonotic disease caused by protozoa, easily disseminated by ingestion of cysts in water and food. For the identification of these parasitological agents, the coproparasitological laboratory techniques are prioritized, being some of them the method of Faust and the technique of Hoffman, Pons and Janner (HPJ). The objective of this study was to evaluate samples of feces from dogs, collected on the shore of the Laranjal beach, county of Pelotas, Rio Grande do Sul, Brazil, and to compare these two diagnostic techniques, used in laboratory routine. Of the 41 samples analyzed by both techniques, nine were positive by the method of Faust and seven by HPJ, representing $22 \%$ and $17 \%$ respectively. Because it is a public place and giardiosis has zoonotic potential, it is necessary to adopt sanitary measures aimed at reducing the contamination of these places and consequently the exposure to this protozoan.
\end{abstract}

Key words: comparison of techniques, diagnostic, giardiasis, protozoan, zoonosis 


\title{
Frecuencia de giardia spp. en fezes de perros en la playa del Laranjal, Pelotas-RS y estudio comparativo entre técnicas
}

\begin{abstract}
RESUMEN. La giardiosis es una importante enfermedad zoonótica causada por protozoarios, fácilmente diseminada por la ingestión de quistes en el agua y el alimento. Para identificación parasitológica de este agente se prioriza las técnicas de laboratorio coprológicas, siendo algunas de ellas el método de Faust y la técnica de Hoffman, Pons y Janer (HPJ). El objetivo de este estudio fue evaluar muestras de heces de perros, recogidas en la orilla de la playa de Laranjal, en el municipio de Pelotas, Rio Grande do Sul, Brasil, y comparar estas dos técnicas de diagnóstico, utilizadas en la rutina de laboratorio. De las 41 muestras analizadas por ambas técnicas, nueve fueron positivas por el método de Faust y siete por la de HPJ, representando el $22 \%$ y el $17 \%$ respectivamente. Pelo fato de ser un local público y la giardiosis presentar potencial zoonótico, es necesario adoptar medidas sanitarias que apunte a la disminución de la contaminación de estos locales y consecuentemente la exposición a este protozoario.
\end{abstract}

Palabras clave: comparación de técnicas, diagnóstico, giardiasis, protozoario, zoonosis

\section{Introdução}

O percentual de doenças diagnosticadas, causadas por parasitos, vem significativamente aumentando, havendo maiores pesquisas na área. A giardiose é uma enfermidade causada pelo protozoário gastrointestinal Giardia spp. pode acometer os hospedeiros levando a dores abdominais, vômitos, diarreia mucosa e, ou, sanguinolenta, intermitente, fator que pode vir a dificultar o diagnóstico (Almeida et al., 2007, Tilley et al., 2008). Em ambientes como praças e praias existem relatos de pesquisas que mostram que há uma grande disseminação tanto de doenças causadas por helmintos quanto por protozoários (Silva et al., 2007). Após instalação no ambiente, os cistos ficam viáveis por bastante tempo, levando em conta condições como temperatura, umidade, disseminação e saneamento básico (Mascarini, 2003).

A transmissão ocorre pela ingestão de cistos, forma infectante, em alimentos ou na água. Este quadro parasitário pode ocorrer em animais domésticos, silvestres e no homem. Em locais onde os cães vivem aglomerados e sem tratamento, existe a facilitação da disseminação. Também é uma doença muito comum em clínicas veterinárias, em animais jovens e podendo ser persistente ou, até mesmo em alguns casos, assintomática (Beck et al., 2005).

Devido a seu potencial zoonótico, desde 2004, a giardiose foi incluída no rol das doenças tropicais negligenciadas, delimitado pela Organização Mundial de Saúde (OMS), grupo este, composto por diversos tipos de agentes, como bactérias, vírus e protozoários (Savioli et al., 2006). Embora, a principal forma de transmissão de giardiose para os humanos, seja a forma direta, ou seja, de pessoa para pessoa, animais domésticos, como cães e gatos podem abrigar espécies do protozoário, infectantes para os humanos (Robertson et al., 2000). Análises moleculares têm demonstrado o mesmo genótipo de Giardia spp. presente em humanos e em outras espécies de mamíferos (Thompson et al., 2000). A principal forma de diagnóstico laboratorial da giardiose é o exame coprológico, dentre estes, podemos citar o exame direto e a hematoxilina férrica em fezes liquefeitas. Em fezes de consistência sólida, o método de Faust é o mais indicado (Brito \& Bastos, 2006). Bica et al. (2011) citam que o exame de sedimentação espontânea em água ou Lutz (1919) e Hoffman et al. (1934) também pode ser utilizado no diagnóstico de giardíase.

Este estudo foi realizado, com o intuito de avaliar a presença de cistos de Giardia spp. em amostras de fezes de cães, presentes na praia do Laranjal, bem como comparar a sensibilidade de duas técnicas utilizadas para diagnóstico desta parasitose.

\section{Material e Métodos}

Neste estudo, foram coletadas, 41 amostras de fezes de cães ao longo da orla da praia do laranjal, no município de Pelotas- RS, Brasil. Todas as amostras foram coletadas em sacos plásticos individuais e acondicionadas em caixas isotérmicas contendo gelo biológico e enviadas ao laboratório. As análises foram realizadas no Laboratório de Doenças Parasitárias (LADOPAR) da Faculdade de Veterinária, da Universidade Federal de Pelotas (UFPel). Na coleta, 
priorizaram-se fezes frescas para evitar resultados falsos negativos.

As técnicas utilizadas para pesquisa de cistos de Giardia spp. nas amostras de fezes, foram a de Faust, elaborada por Faust et al. (1938), que consiste na centrifugo flutuação em sulfato de zinco a $33 \%$, com densidade de $1,18 \mathrm{~g} / \mathrm{ml}$, utilizada para pesquisa de cistos de protozoário e ovos leves de helmintos. A amostra deve ser centrifugada no mínimo duas vezes, sendo que para melhorar a identificação dos cistos de protozoários, o recomendado é a centrifugação de três a quatro vezes, durante um minuto, clarificando significativamente a amostra. Após a última centrifugação, coloca-se em suspensão no meio de flutuação, deixando o tubo em repouso por aproximadamente cinco minutos, recolhendose o sobrenadante com a alça de platina.

Outra técnica utilizada foi a de Hoffman et al. (1934), que utiliza o fundamento da sedimentação espontânea em água, para pesquisa de ovos pesados de helmintos e cistos de protozoários, onde as amostras fecais com aproximadamente 10 gramas, serão filtradas. O sedimento ficará contido na base do cálice, onde a água destilada deverá ser trocada a cada 15 a 20 minutos, repetindo este processo inúmeras vezes até ocorrer a clarificação da água. Ao final do processo, pipeta-se uma amostra do sedimento e coloca-se uma gota em uma lâmina, corando esta gota com lugol para facilitar a visualização dos cistos ao colocar a lamínula $\mathrm{O}$ diagnóstico foi baseado na presença de cistos e/ou trofozoítos de Giardia spp. nas fezes

\section{Resultados}

Das 41 amostras analisadas, nove foram positivas para cistos de Giardia spp. pela técnica de Faust e sete pela de Hoffmann, Pons e Janer (HPJ) (Tabela 1), representando $22 \%$ e $17 \%$ do total de amostras, respectivamente. A análise dos resultados demonstra uma maior sensibilidade da técnica de Faust em relação a técnica de HPJ para detecção de cistos de Giardia spp.

Tabela1. Frequência de Giardia spp, em amostras de fezes de cães, coletadas na praia do laranjal, comparando as técnicas de Faust e de Hoffmann, Pons e Janer.

\begin{tabular}{lccc}
\hline Técnica & Total Amostras & Positivas & $\%$ \\
\hline Faust & 41 & 9 & 22 \\
HPJ & 41 & 7 & 17 \\
\hline
\end{tabular}

\section{Discussão}

Das 41 amostras coletadas e analisadas pela técnica de Faust et al. (1938), 22\% continham cistos de Giardia spp. Utilizando esta mesma técnica para diagnóstico, Beck et al. (2005) encontraram positividade de $34,04 \%$, analisando a frequência de infecção por Giardia lamblia em cães no município de Canoas (RS), resultado semelhante ao encontrado por Bartmann \& Araújo (2004) que avaliando cães em Porto Alegre (RS), encontrou $37,6 \%$ de amostras positivas.

Comparando as duas técnicas, assim como o presente estudo, Bica et al. (2011) observaram que a técnica de Faust et al. (1938) foi mais sensível para a observação de cistos de Giardia spp. do que a de HPJ, pois avaliando 163 amostras fecais de humanos e comparando estas técnicas, encontraram 11 amostras positivas pelo método de Faust et al. (1938) e seis pela de HPJ.

Cognialli et al. (2016) avaliando o limiar de positividade dos métodos de Faust e de sedimentação espontânea para detecção de cistos de Giardia spp. detectou que o método de Faust et al. (1938) foi mais sensível para a detecção de cistos de Giardia duodenalis (76,3\%) em relação ao método de sedimentação $(37,5 \%)$.

A partir dos resultados obtidos, evidencia-se que o método de Faust et al. (1938) trata-se de uma técnica mais segura e eficaz no diagnóstico de helmintos e protozoários em pequenos animais, comparando com a técnica de HPJ (Silva et al., 2007).

Pelo fato de conseguir apresentar maior positividade para cistos de Giardia spp., a técnica de Faust é considerada, em geral, a melhor técnica para identificação deste protozoário, podendo ser utilizada na rotina laboratorial (Bartmann \& Araújo, 2004). Esta superioridade da técnica de Faust et al. (1938) está de acordo com escassos relatos da literatura incluindo a parasitologia veterinária (Gates \& Nolan, 2009).

\section{Conclusão}

De acordo com os resultados obtidos, concluise que existe um grande risco de contaminação de pessoas e animais por Giardia spp na orla da praia do laranjal. Por tratar-se de uma zoonose, é importante adotar medidas sanitárias que visem reduzir a contaminação do ambiente e consequentemente, os riscos de infecção por este protozoário, que está associado a saneamento básico deficiente. 
É importante orientar os proprietários sobre controle do acesso dos animais à rua, fornecer água e alimento de boa qualidade, orientar sobre vermifugação, informando o melhor cronograma para cada categoria animal. Em caso de animais positivos, instituir o tratamento ideal para esta parasitose, controlando, desta forma, a eliminação de cistos no ambiente, diminuindo sua contaminação.

$\mathrm{O}$ encaminhamento de amostras para exame coproparasitológico em caso de animais com suspeita de giardiose é uma ferramenta importante para confirmar o diagnóstico e proceder ao melhor tratamento.

A partir deste estudo, foi possível concluir ainda que a técnica de Faust é a mais indicada para diagnóstico de Giardia spp em laboratório.

\section{Referências Bibliográficas}

Almeida, F. M., Silva, M. M. O. \& Labarthe, N. 2007. Giardia spp. em amostras fecais de gatos domésticos do Rio de Janeiro. Acta Scientiae Veterinariae, 35, 468-469.

Bartmann, A. \& Araújo, F. A. P. D. 2004. Frequência de Giardia lamblia em cães atendidos em clínicas veterinárias de Porto Alegre, RS, Brasil. Ciência Rural, 34, 10931096.

Beck, C. A., Araujo, A. P., Olicheski, A. T. \& Breyer, A. S. 2005. Frequência da infecção por Giardia lamblia avaliada pelo método de Faust e Cols (1939) e pela coloração da Auramina, no município de Canoas, RS, Brasil. Ciência Rural, 35, 126-130.

Bica, V. C., Dillemburg, A. F. \& Tasca, T. 2011. Diagnóstico laboratorial da giardiose humana: comparação entre as técnicas de sedimentação espontânea em água e de centrifugo-flutuação em solução de sulfato de zinco. Revista HCPA, $39-4$.

Brito, T. \& Bastos, A. 2006. Diagnóstico laboratorial da Giardia lamblia. Saúde e Ambiente em Revista, 1, 12-25.

Cognialli, R. C. R., Haidamak, J., Vayego, S. A. \& Klisiowicz, R. D. 2016. Positivity threshold and sensitivity of methods of Faust et al. and Lutz for detection of Giardia duodenalis cysts. Revista Brasileira de Análises Clínicas, 49, 100-104.

Faust, E. C., D'antoni, J. S., Odom, V., Miller, M. J., Peres, C., Sawitz, W., Thomen, L. F., Tobie, J. \& Walker, H. A. 1938. A critical study of clinical laboratory technics for the diagnosis of protozoan cysts and helminth eggs in feces I. Preliminary communication. American Journal of Tropical Medicine, 18, 169-183.

Gates, M. C. \& Nolan, T. J. 2009. Comparison of passive fecal flotation run by veterinary students to zincsulfate centrifugation flotation run in a diagnostic parasitology laboratory. Journal of Parasitology, 95.

Hoffman, W. A., Pons, J. A. \& Janer, J. L. 1934. Sedimentation concentration method in Schistosomiasis mansoni. The Puerto Rico Journal of Public Health and Tropical Medicine, 9, 283-298.

Lutz, A. O. S. m. e. a. S., segundo observações feitas no Brazil. Memórias do Instituto Oswaldo Cruz, 11, 121-55. 1919. Schistosomum mansoni e a Schistomatose, segundo observações feitas no Brazil. Memórias do Instituto Oswaldo Cruz, 11, 121125.

Mascarini, L. M. 2003. A historical approach of the trajectory of the parasitology. Ciências $e$ Saúde Coletiva, 8, 809=814.

Robertson, I. D., Irwin, P. J., Lymbery, A. J. \& Thompson, R. C. A. 2000. The role of companion animals in the emergence of parasitic zoonoses. International Journal for Parasitology, 30, 1369-1377.

Savioli, L., Smith, H. \& Thompson, A. 2006. Giardia and Cryptosporidium join the 'Neglected Diseases Initiative'. Trends in Parasitology, 22, 203-208.

Silva, A. S., Ceolin, L. V., Carganelutti, J. F., Pessoa, G. A., Oliveira, C. B., Quintal, A. P. N. \& Monteiro, S. G. 2007. Prevalência de parasitismo em cães domiciliados num bairro de Santa Maria-RS. Saúde, 33, 27-31.

Thompson, R. C. A., Hopkins, R. M. \& Homan, W. L. 2000. Nomenclature and genetic groupings of Giardia infecting mammals. Parasitology Today, 16, 210-213.

Tilley, L. P., Smith, J. R. \& Francis, W. K. 2008. Consulta veterinária em 5 minutos: Espécies canina e felina. Editora Manole, São Paulo.

\section{Article History:}

Received 17 November 2017

Accepted 5 January 2018

Available online 13 February 2018

License information: This is an open-access article distributed under the terms of the Creative Commons Attribution License 4.0, which permits unrestricted use, distribution, and reproduction in any medium, provided the original work is properly cited. 